\title{
Time-Resolved PIV investigations of the flow field around cod-end net structures
}

\author{
Elkhadim Bouhoubeiny ${ }^{\mathrm{a}, \mathrm{b}}$, Grégory Germain ${ }^{\mathrm{a}, \mathrm{c}}$ and Philippe Druault ${ }^{\mathrm{b}, ~ *}$
}

\author{
a IFREMER, Hydrodynamic \& Metocean Service, 150 Quai Gambetta, 62321 Boulogne-sur-Mer, \\ France \\ ${ }^{\mathrm{b}}$ University Pierre et Marie Curie-Paris 6, Institut Jean Le Rond d'Alembert, CNRS UMR 7190, case \\ 162, 75252 Paris Cedex 5, France \\ ${ }^{c}$ University Lille Nord de France, F-59000 Lille, France
}

* Corresponding author : Philippe Druault, email address : philippe.druault@upmc.fr

\begin{abstract}
:
Flow field measurements past a fixed rigid cod-end structure and past a porous fishing net structure are conducted using Time-Resolved PIV method. The rigid cod-end is first used to characterize finely the wake flow. Proper Orthogonal Decomposition (POD) is then applied in order to extract the large scale energetic vortices of the flow from the measured velocity field. It is then observed that the first POD modes are associated to the Karman's type flow structure of vortex shedding. It is shown that the characteristics of the wake flow behind the rigid cod-end flow configuration compare quite well with previous ones obtained from bluff cylinder or sphere wake analyzes. Second, PIV measurements are performed around a non-rigid bottom trawl which is free to move. Preliminary analyses show that the frequencies associated with the oscillatory motion of the porous structure are the same as the ones detected in the near wake flow demonstrating the lock-in regime. It is then expected that these preliminary results provide some interesting informations about future investigations on the drag force acting on fishing net structure.
\end{abstract}

Keywords: Fishing gear; PIV measurements; Vortex shedding; Proper Orthogonal Decomposition

\section{Introduction}

The analysis of hydrodynamics of various types of fishing net structures, and especially of a trawl, has been of great interest for scientists for a long time. Such investigation has an impact not only on commercial fishing operations including the fishing vessel energy e_ciency but also on biological and socio-economical environment. For bottom and pelagic trawls, the hydrodynamic turbulent flow has a great influence i) on the stability of the movement of the fishing net structure; ii) on the drag force of the fishing gear and iii) on selectivity (the ability of fishing gears to prevent non-target fish catches). Due to its impact on fuel consumption, the second point is more and more crucial for fishermen and researchers [1,2]. The knowledge of these drag forces allows then the definition of the shape and the behaviour of the structure during a trawling process, and tension and loads in its threads and ropes [3]. Globally, it is now well known 
that most of the fuel consumed during a fishing trip is used to tow the fishing gear and also that the trawls are responsible of the largest part of the fuel consumption during fishing operations. But, the determination of the drag force acting on net structures is a complex task. Even if the measurement of the whole drag force on the net structure with different size of catches $[4,5]$ may be possible, the determination of the drag contribution associated with each part of the trawl (net, catch, hydrodynamic forces...) remains today inconceivable. In this sense, we propose in this work to only focus on the hydrodynamic flow around the net structure hoping that a better understanding of the flow behaviour will provide some relevant information for future analyses of the drag force.

Porous structure poses challenging problem for the understanding of bluff body wakes. Indeed, porous structures are quite different to the usual classical rigid bluff bodies such as cylinders, spheres or disks which have been extensively analyzed in the past (see for instance the reviews by $[6,7])$. Furthermore, due to the flexibility of the net, there is a complex interaction between the flow, the shape of the net and its behaviour. In fact, the analysis of the hydrodynamic flow around (and also in) fishing net structures is extremely related to the strong influence of hydrodynamic fields on the shape of trawl elements, acting forces, fish behaviour and on catchability of fishing gears. The variations of incidence on the different part of the net (from zero to ninety degrees) induce different kind of behaviour of the flow, from laminar flow with a boundary layer development on the horizontal part of the net to separate flow through a porous structure for the higher panel angles or behind specific parts like the cod-end.

Despite its practical engineering application, there is almost no reported study dealing with the hydrodynamic flow analysis around porous structure and especially around realistic porous trawl free to move in the flow. Yet, it is clear that hydrodynamic flow has a very significant influence on the trawl moving process. To investigate the flow in presence of a net and a catch, Pichot et al. [8] previously used Laser Doppler Velocimetry (LDV) measurements to study the mean flow field over a rigid cod-end with a closed and an open net entrance. Other previous works have focused on the analysis of different mesh sizes or numbers to improve the selectivity without taking into account the hydrodynamic flow. Instead of focusing on the hydrodynamic flow analysis, previous experimental works proposed to determine the total drag force using empirical formulations based on numerous assumptions [9]. Besides, the complexity of the flow makes numerical simulations difficult, even if several authors made significant advances [10]. Today no effective model exists for such investigation [11]. An important work should be done on this area to improve the knowledge of the hydrodynamic flow past a fishing net structure.

It is important to predict the flow motion accurately: alternate shedding of vortices, which develops in the near wake, causes pressure forces which generate structural vibration and drag forces. In this sense, an experimental investigation is conducted to study the near wake of the flow around a cod-end, considered here as one of the more important part of a trawl (see figure 1). To simplify the issue, we firstly propose to investigate a modelled rigid cod-end net structure. Secondly, a 1/10 scaled bottom trawl is considered. In this last flow configuration, the fishing net structure is free to move. Particle Image Velocimetry (PIV) is implemented to access the instantaneous velocity fields in each flow configuration. To investigate the large scale flow structures around the cod-end, specific mathematical post-processing procedure such as Proper Orthogonal Decomposition is applied. Part 2 of the paper is devoted to the experimental set up and measurement method. Part 3 deals with the mathematical post-processing tool used to analyze PIV database. Then after presenting global flow results, the large scale flow structure characteristics in the wake of both rigid and free to move cod-end are examined. 


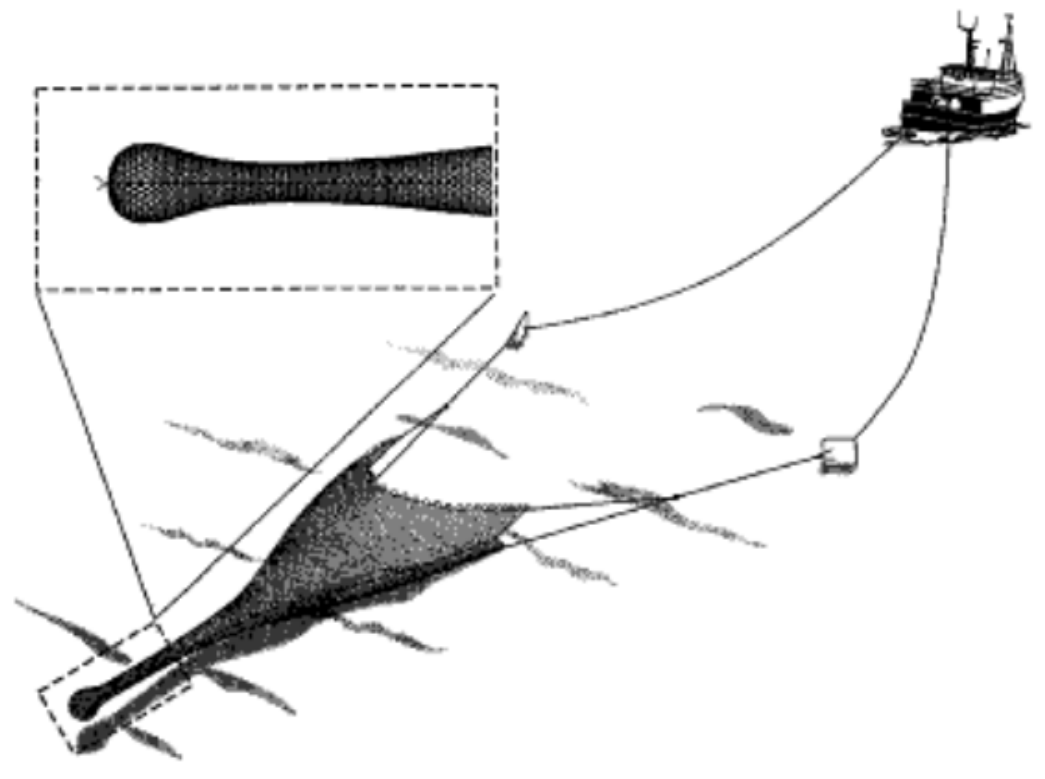

Figure 1: Bottom trawl showing the position of the cod-end. 


\section{Experimental apparatus}

\subsection{Flume tank and fishing gear}

Experimental campaigns are performed in the IFREMER (French Research Institute for Exploitation of the Sea) wave and current circulation flume tank shown in figure 2 . The dimensions of the flume tank are $18 \mathrm{~m}$ (length) $\times 4 \mathrm{~m}$ (width) $\times 2 \mathrm{~m}$ (depth). A side observation window of $8 \mathrm{~m}$ by $2 \mathrm{~m}$ placed on one side of the tank allows users to observe the behaviour of the models during trials and to carry out video sequences. The bottom of the flume is a conveyor belt which can be synchronized with the water speed in order to simulate devices in contact with the bottom, like bottom trawls. The flow turbulence can be adjusted between 5 to $28 \%$ and the flow streamwise velocity range is 0.1 to $2.2 \mathrm{~m} . \mathrm{s}^{-1}$.

The first flow configuration is the rigid cod-end given in figure 3. This rigid cod-end is used in order to avoid flow instabilities due to the motion of fishing gear. The flow around this structure is then axisymmetric. The catch inside the cod-end is limited by two spherical caps of diameter $D=0.454 \mathrm{~m}$. The model is $1 \mathrm{~m}$ long, made of PA twine of $1200 \mathrm{~m} / \mathrm{kg}$, with diamond-shaped meshes of $30 \mathrm{~mm}$ lens.

This rigid structure is placed in a flow with a fixed input streamwise velocity of $U_{\text {ref }}=0.5 \mathrm{~m} \cdot \mathrm{s}^{-1}$ and a turbulence intensity rate of $8 \%$. The corresponding Reynolds number $R e=U_{r e f} D / v$ is then 227000 .

The second flow configuration is a $1 / 10$ scaled bottom trawl. The model bottom trawl located at the bottom of the flume tank, is free to move in the water flow. For such configuration, the coveyor belt is synchronized with the flow speed. The model is $4 \mathrm{~m}$ long, made with diamondshaped meshes of $8 \mathrm{~mm}$ lens. The diameter of the cod-end is $d=0.1 \mathrm{~m}$. The bottom trawl is placed in a flow with a fixed streamwise velocity of $0.55 \mathrm{~m} . \mathrm{s}^{-1}$ with a similar turbulence intensity rate than in the other flow configuration.

\subsection{PIV system}

Time-Resolved PIV (TRPIV) measurement method is used to investigate the velocity field past both fishing net structures. The method that measures of the existing velocities around fishing gear in the flume tank offers two main advantages: (1) it is non-intrusive and (2) it offers the possibility of mapping the velocities on one or more planes, in real time, and under unsteady conditions. This technique is based on illuminating the seeding particles and storing the resulting camera images to analyze displacements of particles between two successive images [12]. For the PIV measurements, the entire volume of water contained in the tank is seeded with glass spheres with an average diameter of $15 \mu \mathrm{m}$. The velocities are obtained by dividing the distance by the elapsed time of laser pulses. In this work, PIV system is implemented to determine the longitudinal $u_{1}$ and transverse $u_{2}$ velocity components along the $x$ (or $x_{1}$ ) and $y$ (or $x_{2}$ ) directions respectively. TRPIV is unique in providing data that can be used to extract not only spatial instantaneous flow structures but also frequencies associated with these flow structures. TRPIV database used for frequency analysis should satisfy the Nyquist criterion: database must then be sampled with at least twice the highest frequency present in the measurement signal. In this sense, we retain an image acquisition rate allowing the time resolved sampling of the vortex shedding phenomenon.

The PIV system consists of a double pulsed laser type two-chamber Gemini PIV Nid-Yag $2 \mathrm{x}$ $120 \mathrm{~mJ}$ at $15 \mathrm{~Hz}$, and a Hi-sense CCD camera. The laser is placed behind the catch of the rigid 


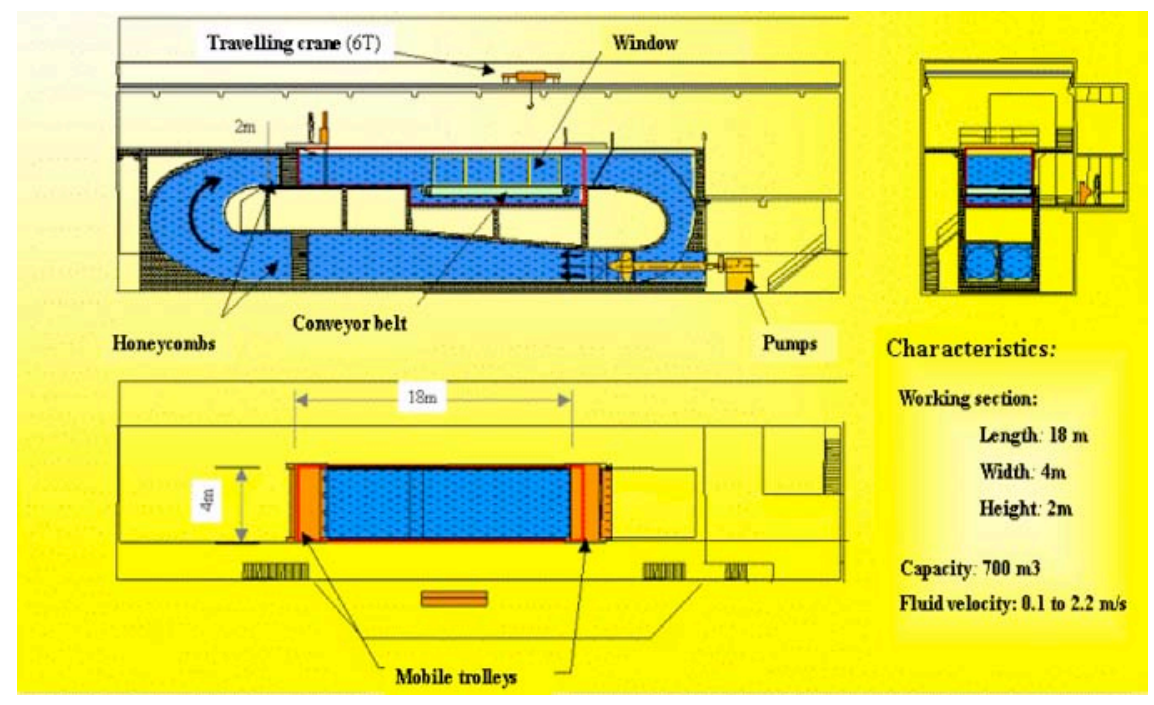

Figure 2: Ifremer free surface hydrodynamic water tunnel.

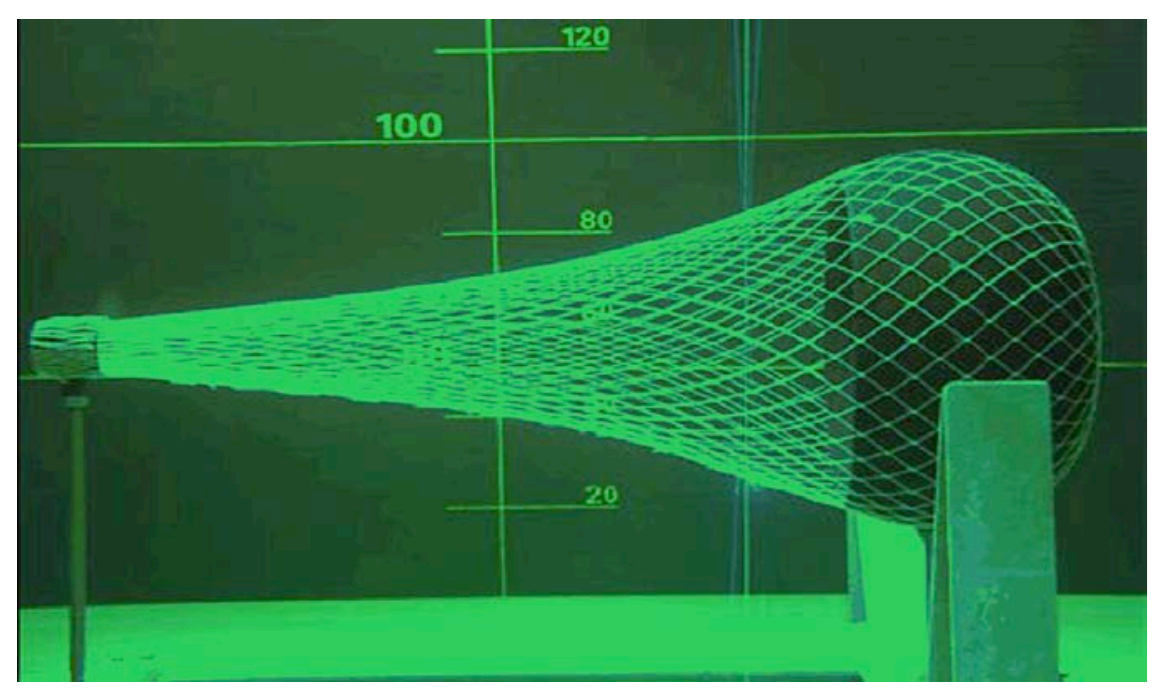

Figure 3: Fixed rigid cod-end structure in the flume tank. 


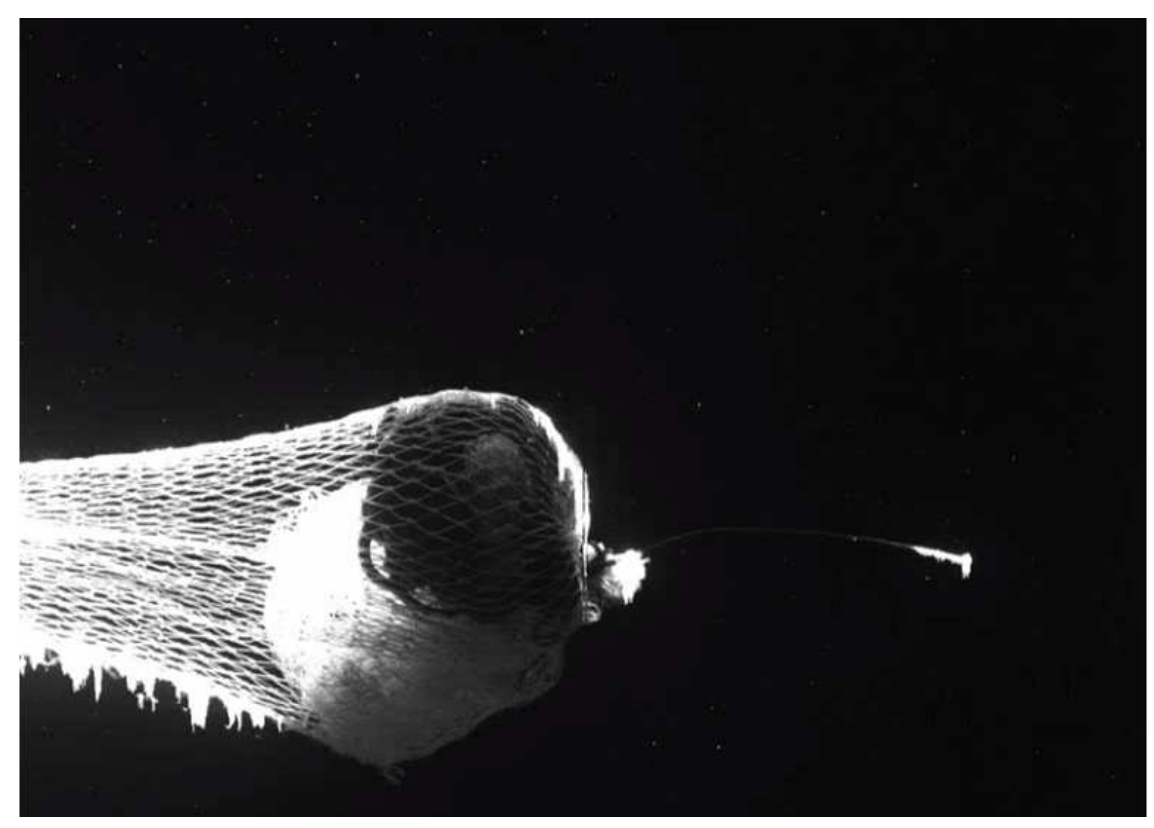

Figure 4: Porous cod-end able to free moving in the flume tank.

cod-end and the camera is outside the tank. Two cameras were used. The first one has a 4 $\mathrm{Hz}$ frequency for a double-frame images with a $1280 \times 1024$ pixels $^{2}$ resolution used for flow measurements around the rigid co-end. The second camera has a $15 \mathrm{~Hz}$ frequency for a doubleframe images with a $1600 \times 1200$ pixels ${ }^{2}$ resolution used for flow measurements around the bottom trawl. The image processing is done with the Flow Map 1500 software from Dantec dynamics. Finally, instantaneous velocity fields are obtained using a cross-correlation PIV algorithm. The interrogation windows size is $32 \times 32$ pixels $^{2}$ and adjacent windows are $25 \%$ overlapped.

\subsection{PIV measurements}

In this article, we mainly focus on the analysis of the flow past the rigid cod-end. Only preliminary TRPIV results deduced from the second flow configuration (bottom trawl) will be presented.

Rigid cod-end flow configuration.

To investigate the near wake behind the rigid cod-end net structure, PIV system is implemented to determine the velocity field vectors in different planes. The longitudinal $u_{1}$ and transverse $u_{2}$ velocity components are then measured around the cod-end. In order to obtain a good description of the wake, two measurement campaigns using different PIV system parameters are successively performed.

The first one referred to PIV database 1 for the rest of the paper, uses a focal lens length of the camera of $60 \mathrm{~mm}$. The size of the image is $810.7 \times 648.5 \mathrm{~mm}^{2}$ corresponding to $1280 \times$ 1024 pixels $^{2}$. Two series of 190 successive velocity fields are obtained in the plane located in the near wake of the rigid cod-end (see figure 5) at a frequency of $4 \mathrm{~Hz}(0.25 \mathrm{~s})$. In this plane, instantaneous velocity fields are obtained on a mesh of $n_{x} \times n_{y}=42 \times 53=2226$ points. The 


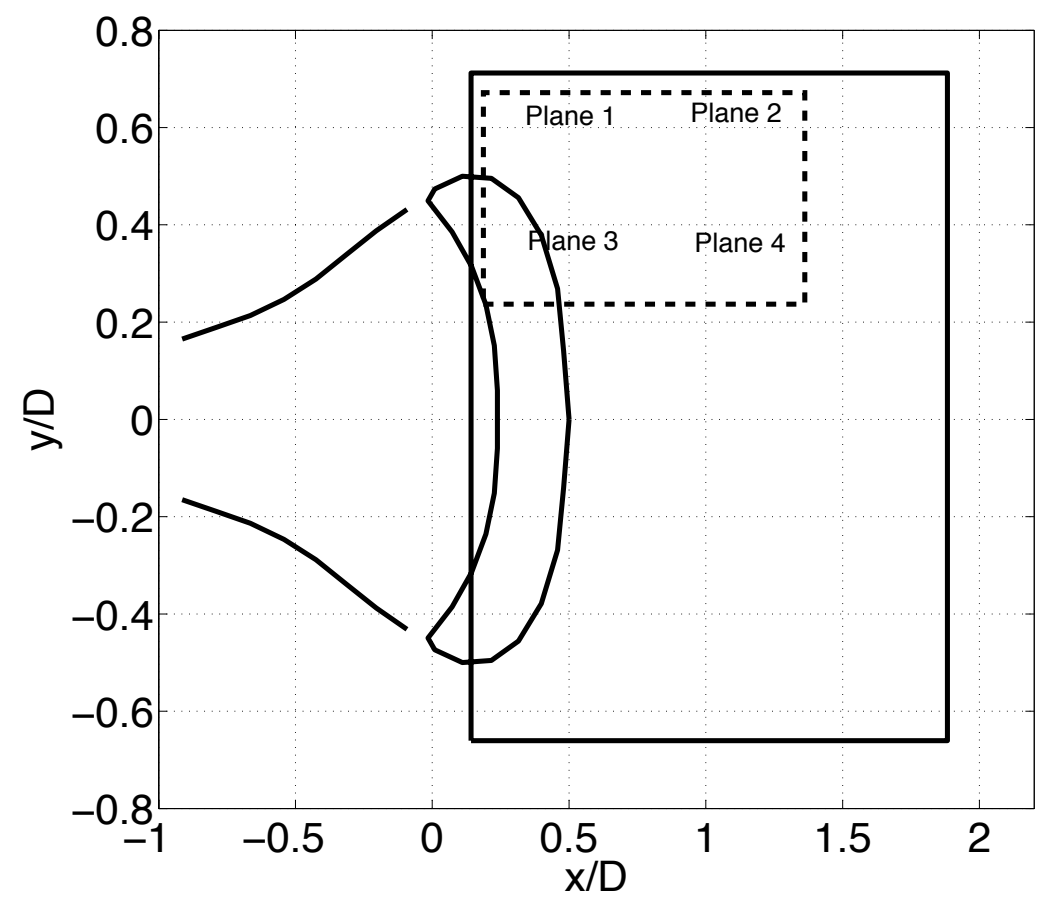

Figure 5: Description of PIV measurements plane. Black line box corresponds to the first PIV measurement campaign. Dash-Line box corresponds to the space domain investigated in the second PIV measurement campaign.

distance between two consecutive mesh points is $15.2 \mathrm{~mm}$ in each direction.

In the second campaign which will be referred to PIV database 2 for the rest of the paper, measurements were obtained with a focal lens length of the camera of $20 \mathrm{~mm}$, in four selected planes. These planes which overlap each other are included in the dash line box given in figure 5 and the size of each plane is $387.9 \times 145.4 \mathrm{~mm}^{2}$. The time between two PIV images is always $0.25 \mathrm{~s}(4 \mathrm{~Hz})$. 500 instantaneous PIV velocity vector fields are obtained on a mesh of $n_{x} \times n_{y}=66 \times 24=1584$ points, in each measurement plane. The spatial resolution is $5.5 \mathrm{~mm}$ in both directions.

Bottom trawl flow configuration.

In the following, only preliminary TRPIV measurements are provided. In this flow configuration, the laser is placed above the bottom trawl and the camera is outside. Only one measurement plane is considered. This measurement will be referred to PIV database 3. 272 instantaneous PIV images are then obtained on a regular mesh $(d x=d y=5.45 \mathrm{~mm})$ of $n_{x} \times n_{y}=66 \times 49=3234$ points, in the selected plane along the bottom trawl (see figure 6). The time between two images is $0.067 \mathrm{~s}(15 \mathrm{~Hz})$. On this figure, two trawl positions corresponding to the bottom position and the top one respectively are also provided. Such representation clearly demonstrates the free movement of the trawl, not only in the transverse $y$-direction but also in the longitudinal $x$ direction (and also in the third direction that can not be visualized from 2D measurements). 


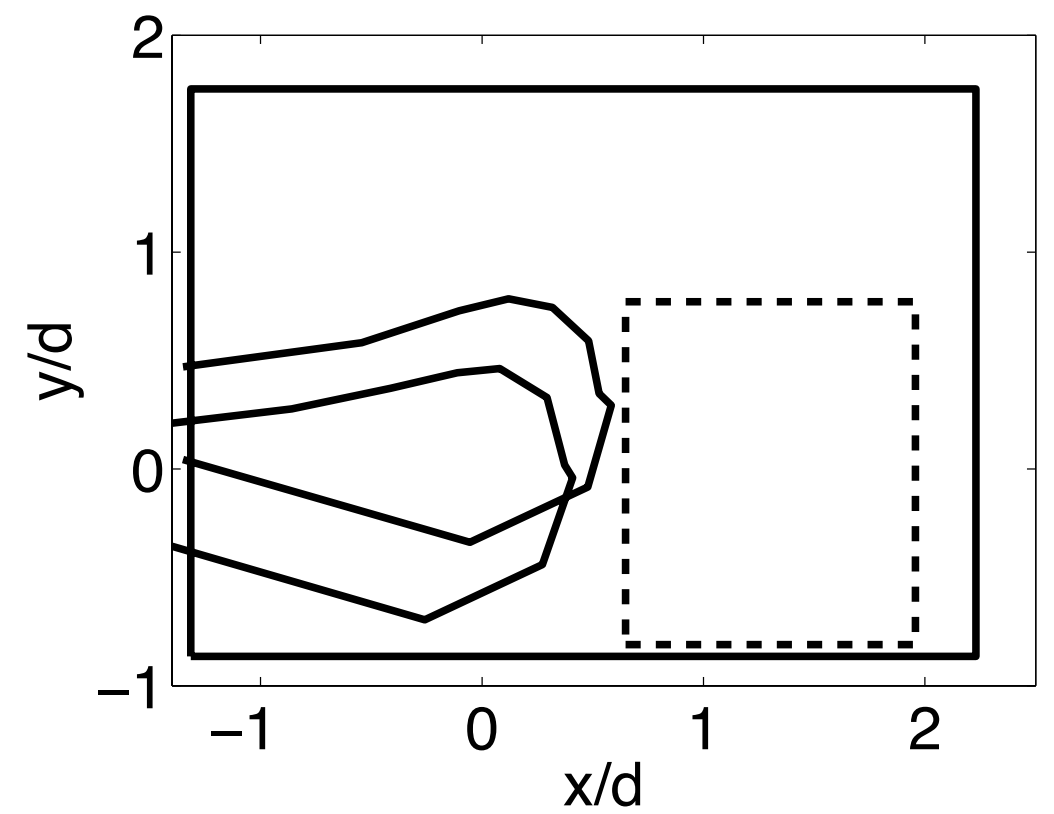

Figure 6: Description of PIV measurements plane. Black line box correspond to the measurement plane. Dash-line box correspond to the space domain further investigated with POD procedure.

\section{Proper Orthogonal Decomposition (POD) mathematical tool}

The Proper Orthogonal Decomposition (POD) which appears under several other names as well (Singular Value Decomposition (SVD), Karhunen Loeve Decomposition (KLD), Principal Component Analysis (PCA)) is a powerful and elegant method for random data analysis. In fluid mechanics and especially for turbulent flow analysis, POD technique has become more and more a universal mathematical tool. Indeed, a relatively small number of POD eigenfunctions allows the extraction of the dynamical behavior of the organized large-scale flow structures present in turbulent flow. Using this property, POD has been also intensively used to formulate a low order dynamical system [13]. Furthermore, POD procedure can be also used as an advanced mathematical tool for the reconstruction of missing experimental data, not only in the space domain $[14,15,16]$ but also in the temporal domain [17]. After recalling the mathematical concept of the POD, its application from 2D PIV database is presented.

\section{Mathematical concept of POD.}

The application of Proper Orthogonal Decomposition to turbulent flow analysis was first proposed by Lumley in 1967 [18] as an objective method to identify deterministic features in turbulent flows. According to Lumley [18], an organized flow structure called coherent structure is the structure that has the largest mean square projection of the velocity field. Mathematically, it corresponds to the determination of a basis which is optimal in an energetic sense. It consists of searching a function $\phi(\mathbf{X})$ which maximizes in a mean square sense the projection of the instantaneous fluctuating velocity field on $\phi(\mathbf{X})$. This maximization leads to a Fredholm integral eigenvalue problem [13] whose kernel is the time averaged two-point spatial correlation tensor 
of the fluctuating velocity components $\left(u_{i}, u_{j}\right): R_{i j}\left(\mathbf{X}, \mathbf{X}^{\prime}\right)=\overline{u_{i}(\mathbf{X}) u_{j}\left(\mathbf{X}^{\prime}\right)}$ where $\mathbf{X}$ indicates the vector space variable and an overbar corresponds to the time averaged operation. The integral eigenvalue problem is:

$$
\int_{\mathcal{D}} R_{i} j\left(\mathbf{X}, \mathbf{X}^{\prime}\right) \phi_{j}\left(\mathbf{X}^{\prime}\right) d \mathbf{X}^{\prime}=\lambda \phi_{i}(\mathbf{X})
$$

where $\phi_{i}$ denotes the spatial orthogonal eigenfunctions associated with the $u_{i}$ velocity component, $\lambda$ is its corresponding eigenvalue and $\mathcal{D}$ is the spatial domain under investigation.

If the field has finite total energy, Hilbert Schmidt theory assures that the solution exists and consists of a denumerable set of eigenvalues, $\lambda^{n)}$, and corresponding eigenfunctions $\phi^{(n)}$. The modes are ordered so that the first mode contains most of the energy and the relation between the kinetic energy $\left(k=\overline{u_{1}^{2}}+\overline{u_{2}^{2}}\right)$ and the eigenvalues is given by $k=\sum_{n=1}^{N_{\text {mod }}} \lambda^{(n)}$, where $N_{\text {mod }}$ which is the total number of POD modes corresponding to the dimension of the spatial correlation matrix multiplied with the number of velocity components taken into account. Based on this flow decomposition each instantaneous fluctuating velocity field (for instance the $u_{i}$ component) can then be expressed as follows:

$$
u_{i}(\mathbf{X}, t)=\sum_{n=1}^{N_{m o d}} a^{(n)}(t) \phi_{i}^{(n)}(\mathbf{X}),
$$

where $a^{(n)}(t)$ is the $\mathrm{n}^{\text {th }}$ random temporal coefficient of projection of $u_{i}(\mathbf{X}, t)$ onto the $\mathrm{n}^{\text {th }}$ POD eigenfunction $\phi_{i}^{(n)}(\mathbf{X})$. Such previous development describes the classical formulation of the POD procedure introduced by Lumley.

In the Lumley's description, the dimension of the spatial correlation deduced from two-dimensional PIV images is $2 n_{x} \times 2 n_{y}$ where $\left(n_{x} \times n_{y}\right)$ correspond to the number of PIV grid points available on each flow image. Generally when dealing with PIV measurement technique, the databases have a good spatial resolution and a poor temporal one. In this sens, an equivalent POD application introduced by Sirovich [19] is used. This method called snapshot POD proposes to reduce the size of the POD eigenfunction problem in computing the eigenfunctions of the spatial average temporal correlation tensor. Based on 2D PIV velocity fields, this correlation tensor is computed as follows:

$$
R\left(t, t^{\prime}\right)=\frac{1}{2 n_{x} n_{y}} \sum_{i=1}^{2} \sum_{l=1}^{n_{x}} \sum_{m=1}^{n_{y}} u_{i}\left(\mathbf{X}_{l m}, t\right) u_{i}\left(\mathbf{X}_{l m}, t^{\prime}\right)
$$

The integral eigenvalue problem is then determined from this correlation tensor providing temporal orthogonal POD modes $a^{(n)}(t)$. The spatial eigenfunctions are then deduced from the projection of the instantaneous fluctuating velocity field onto $a^{(n)}(t)$. Finally such snapshot flow decomposition provides a similar expression of each fluctuating velocity component (equation 2). Note that both classic and snapshot flow decompositions provide uncorrelated POD temporal coefficients: $a^{(n)} a^{(p)}=\lambda^{(n)} \delta_{n p}$ with $\delta$ the Kronecker symbol and orthonormal POD eigenfunctions satisfying $\phi^{(n)} \phi^{(p)}=\delta_{n p}$.

POD has become a popular method for analyzing turbulent flows and especially the large scale flow structure dynamics [15, 17, 20, 21, 22, 23]. For instance, recently Huera-Huarte and Vernet [23] used POD to reduce the dimensionality of the data for better investigating the wake of an oscillating flexible cylinder. Van Oudheusden et al. [20] and Perrin et al [22] used POD to ob- 
tain phase characterization of vortex shedding in the wake of a square section cylinder and of a circular cylinder respectively.

Application from 2D PIV velocity fields.

In this study, POD procedure is used as a mathematical tool for post-processing PIV velocity field measurements in order to extract the main dynamical features of the flow field behind the rigid cod-end. For such an investigation, the numerical methodology allowing the determination of POD modes is briefly recalled. Similar POD implementation can be found in Druault et al. [17]. Practically, the following steps are taken:

- PIV measurements of the instantaneous velocity fields (two components) in a two-dimensional plane

- Computation of the fluctuating velocity field using the Reynolds Decomposition

- Computation of the temporal two-point correlation tensor for the fluctuating velocity field

- Resolution of the eigenvalue problem (Fredholm equation)

- Projection of the fluctuating velocity field onto some selected first POD modes allowing the extraction of the large scale energetic structures of the flow

\section{Results}

According to the rigid cod-end flow configuration, both measurement campaigns detailed in section 2.3 are successively analyzed. First, a global mean flow analysis is provided. Secondly, the POD technique is applied to educe the spatial large scale flow structures in the wake of the rigid hemispherical structure. Finally, an analysis of the vortex shedding frequency behind the structure is performed. This last analysis is coupled to the POD one.

Note that the following results are presented in a dimensionless version using the diameter $D$ of the rigid or free cod-end structure and the inlet streamiwe velocity $U_{\text {ref }}$ as reference length and velocity respectively.

\subsection{Reynolds averaged field analysis}

The mean velocity flow field $\overline{\mathbf{U}}$ is analyzed according to the Reynolds averaging decomposition. For the PIV database 1, the mean flow field is obtained by averaging 190 average instantaneous velocity fields which correspond to approximately 10 vortex shedding periods. Figure 7 displays the mean flow streamline topology deduced from the first database. This mean flow pattern has two counter rotating vortices which are symmetric along the $y=0$ axis. It is a result of a symmetric pattern of $u_{1}$ and asymmetric one for $u_{2}$. Using this mean flow velocity field, the dimensionless recirculation length $L / D$ is then deduced leading to obtain a value of 1.32 . This value is quasi-similar to the previous ones (between 1.1 and 1.4) computed from flow field past a circular cylinder at high Reynolds flow [24].

Note that both vortices are not perfectly symmetric and for $x / D$ superior to 1.5 the mean flow field has a weak mean $\overline{U_{2}}$ velocity component. This is explained by the experimental device. Indeed, the rigid cod-end trawl is located near the bottom wall of the flume tank, the presence of the wall influencing the mean flow direction.

Using the second PIV database 2 for which four different planes located in the upper part of 


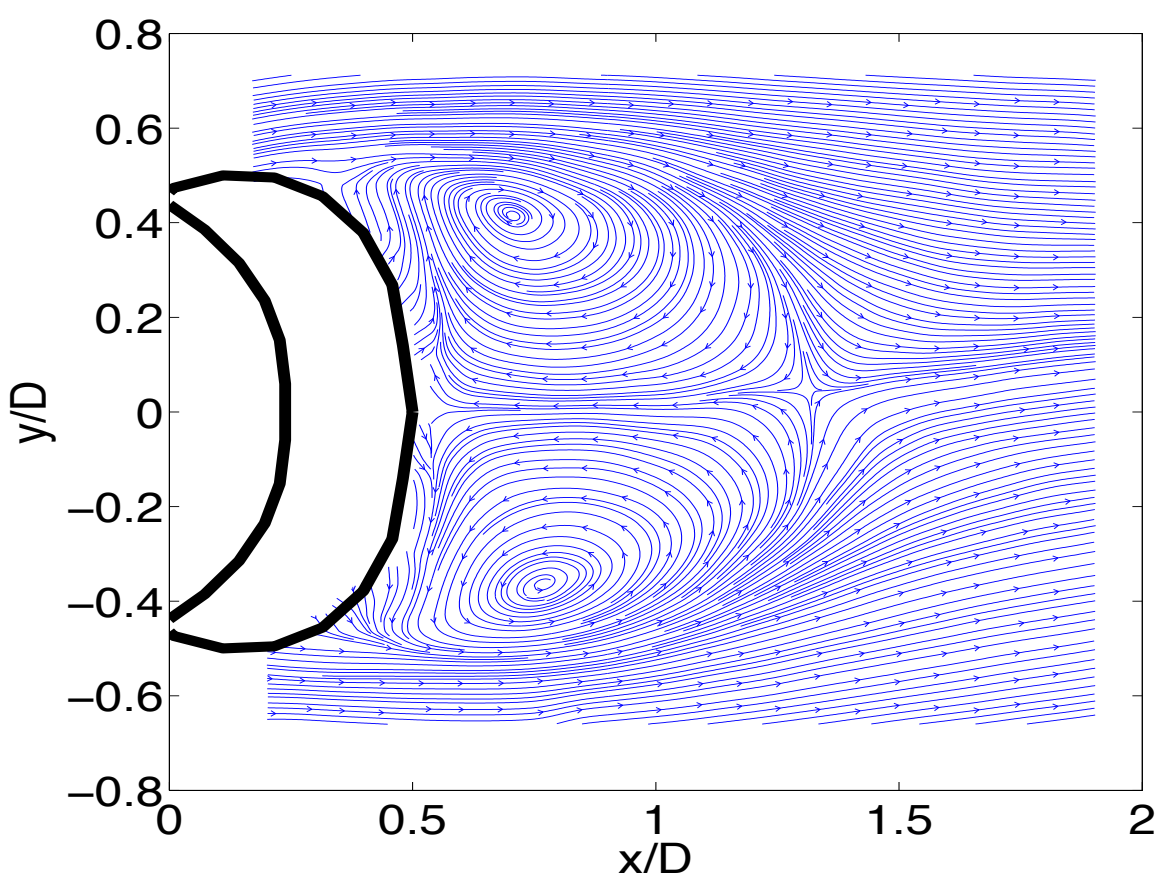

Figure 7: PIV database 1 Mean flow streamline topology. 
the wake are considered, 500 instantaneous fields are used to compute the mean flow field. The time duration of measurements correspond to 25 vortex shedding periods. Figure 8 presents the resulted mean flow field in each available plane. This is in a good agreement with previous ones given on figure 7 (the location of the vortex center is the same in both cases). These results show that the resolution is sufficient for both cases, the global map given the mean flow field in one shot.
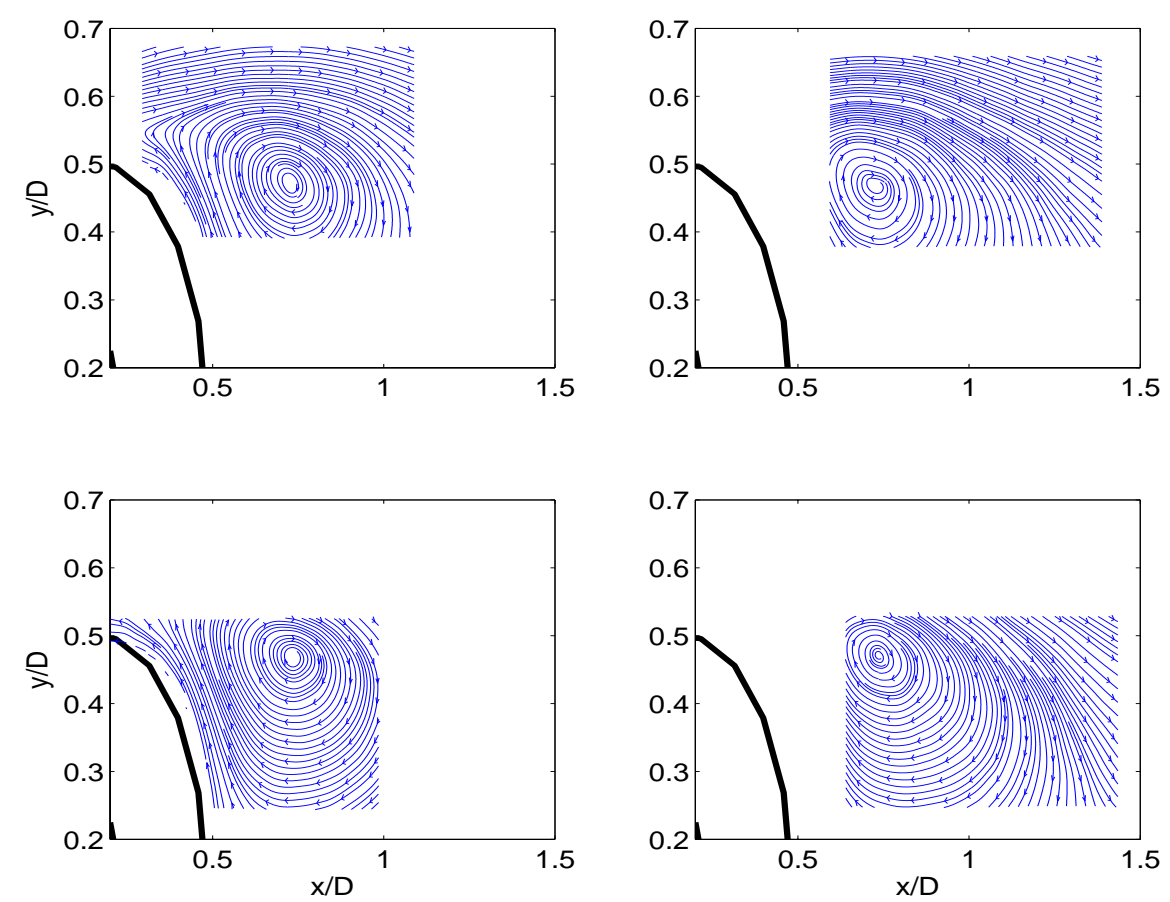

Figure 8: PIV database 2: Mean flow streamline topology.

Figure 9 displays the kinetic energy, $k=\overline{u_{1}^{2}}+\overline{u_{2}^{2}}$, and the Reynolds stress components $\overline{u_{1}^{2}}, \overline{u_{2}^{2}}$ and $\overline{u_{1} u_{2}}$. These variables are normalized with $U_{\text {ref }}^{2}$ and computed from PIV database 1 . Due to a poor correlation during the PIV images in lower left hand corner of the images, affected data are removed. We then observe that both $k$ and $\overline{u_{1}^{2}}$ have the highest activity along the separated shear layer. For the Reynolds transverse component $\overline{u_{2}^{2}}$, the maximum occurs on the wake centerline at approximately 1.3 which approximately around the reattachement point. The representation of stress Reynolds components compares quite well with previous ones deduced from the flow field past a cylinder at $R e=140000$ [24]. When comparing the kinetic energy representation to previous ones, some differences appear due to the fact that in our study, $k$ is only computed from $\overline{u_{1}^{2}}$ and $\overline{u_{2}^{2}}$. The normal Reynolds component $\overline{u_{3}^{2}}$ may have a non-negligible value leading to be taken into account to fully investigate the kinetic energy. Furthermore, the turbulent kinetic energy levels in the upper part of the wake are superior to the ones obtained in the bottom part. 
This is also explained by the experimental device and the presence of the bottom wall justifying the investigation of the wake flow in the upper part of the wake (see PIV database 2). Similar results are obtained when dealing with PIV database 2.

Figure 10 displays the turbulent production $p$ in a dimensionless version. Having a twodimensional database, this term is computed as follows:

$$
p=\overline{u_{1}^{2}} \frac{\partial u_{1}}{\partial x}+\overline{u_{1} u_{2}} \frac{\partial u_{2}}{\partial x}+\overline{u_{1} u_{2}} \frac{\partial u_{1}}{\partial y}+\overline{u_{2}^{\prime 2}} \frac{\partial u_{2}}{\partial y}
$$

Even if PIV mesh grid is not sufficient to well compute the spatial derivatives, it is observed that the maximum values of $p$ coincide with those of the kinetic energy and especially near the shear layer, which is in agreement with previous results based on cylinder wake flow configuration.

\subsection{Proper Orthogonal Decomposition analysis}

Based on each available PIV velocity field (PIV database 1 and 2), a similar procedure is performed to extract the large scale structures of the flow with the aid of POD procedure. The following procedure is detailed at the end of $\S 3$. For each PIV flow field associated with a PIV measurement plane, $N_{t_{1}}=190$ and $N_{t_{2}}=500$ instantaneous fluctuating velocity fields deduced from PIV database 1 and 2 respectively, are used to compute the POD eigenfunctions and corresponding eigenvalues. $N_{\text {mod }_{1}}=190$ and $N_{\text {mod }_{2}}=500$ POD modes are then obtained respectively. Knowing that for the PIV database $1, N_{t_{1}}$ PIV snapshots are only available, the statistical convergence of the first POD modes has to be investigated. The sensitivity of the POD analysis results in relation to the number $N_{t_{1}}$ of available snapshots is studied. The results obtained for the first four eigenvalues are plotted on figure 11 and show that the magnitude of the largest eigenvalues does not change significantly for $N_{t_{1}}$ larger than 160 . This indicates that the number of velocity fields $N_{t_{1}}=190$ is sufficient for providing statistically converged results for at least the first POD modes.

Because the POD modes are the optimal decomposition for the flow (at least for the first two statistical moments), only the first POD modes are needed to capture the main large scale flow structures. High order POD modes are considered to be a part of turbulent fluctuations. Moreover, due the small number of available PIV snapshots (PIV database 1), the use of these high order POD modes for further analysis may be questionable due to a problem of statistical convergence and/or PIV measurement noise.

Figures 12 presents the POD energy convergence deduced from each database. We voluntary limit the $x$-axis to the first values to better appreciate the convergence of the POD eigenvalues. On the left hand side, the evolution of the energy contained in the first POD modes versus the number of POD modes is plotted on a logarithmic scale allowing to demonstrate that the first four eigenvalues almost occur in pair (see table 1).

This is in agreement with many previous studies associated with the analysis of bluff body wake. It is directly related to the particular flow organization where the large scale energetic structures are represented by pairs of spatio-temporal modes [25].

The cumulative energy content is given as a function of the POD mode number in figure 12 (right hand side). For each PIV measurement plane of PIV database 2, POD energy convergence is given. It is then observed that some similar tendencies are observed indicating that the flow organization is globally similar in each measurement plane. This result is linked to the presence of the same large scale rotating flow structure in each plane (see figure 5). A large part of the turbulent kinetic energy is concentrated on the first POD modes. For instance, the first 5 POD modes contains more than $55 \%$ of the total fluctuating kinetic energy. 

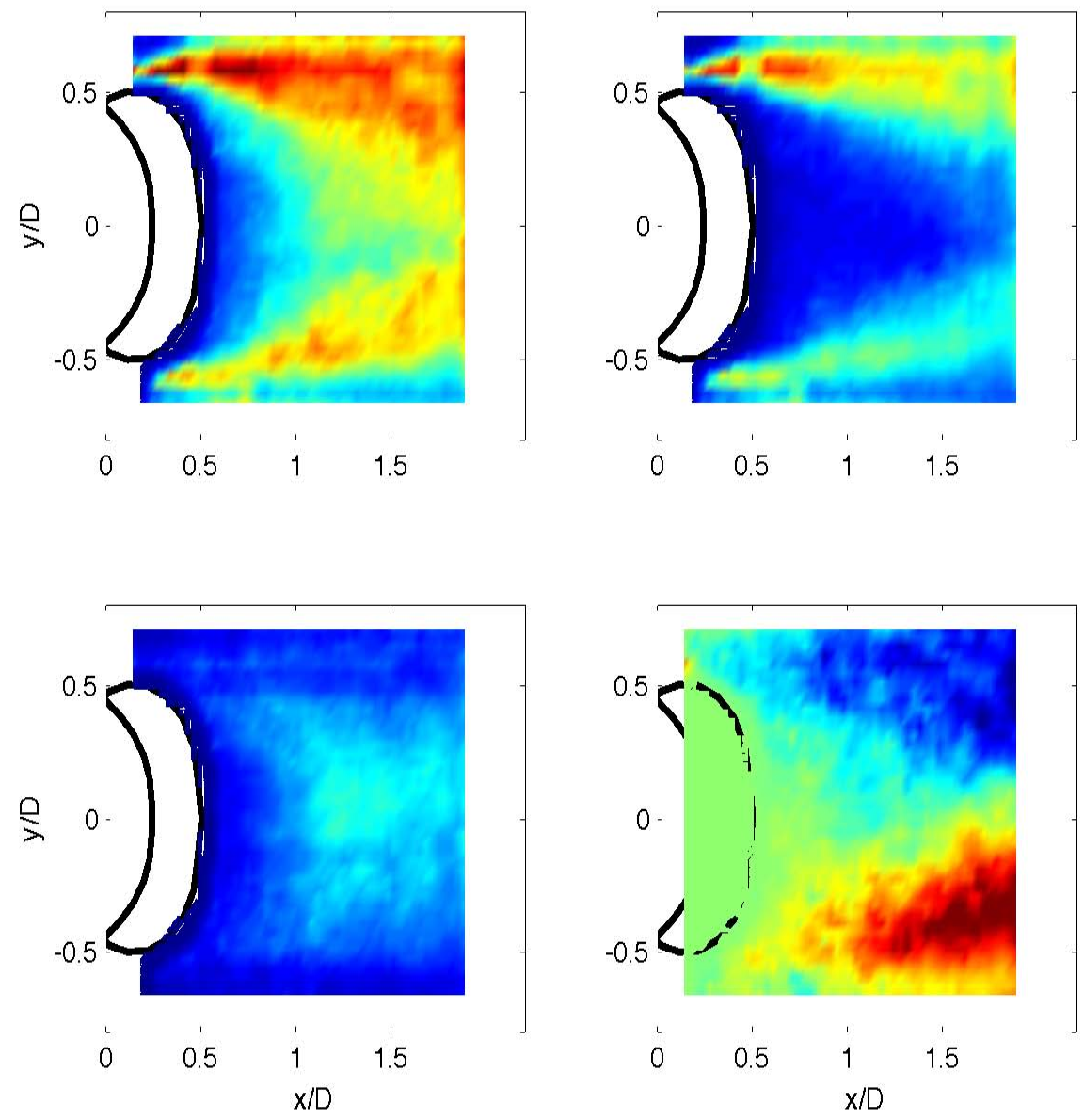

Figure 9: PIV database 1 top: left to right : normalized kinetic energy, Reynolds stress component $\overline{u_{1}^{2}}$ Bottom: left to right normalized Reynolds stress components $\overline{u_{2}^{2}}$ and $\overline{u_{1} u_{2}}$. Colormap ranges from 0 to 0.02 except for $\overline{u_{1} u_{2}}$ which ranges from 0.08 to 0.08 . 


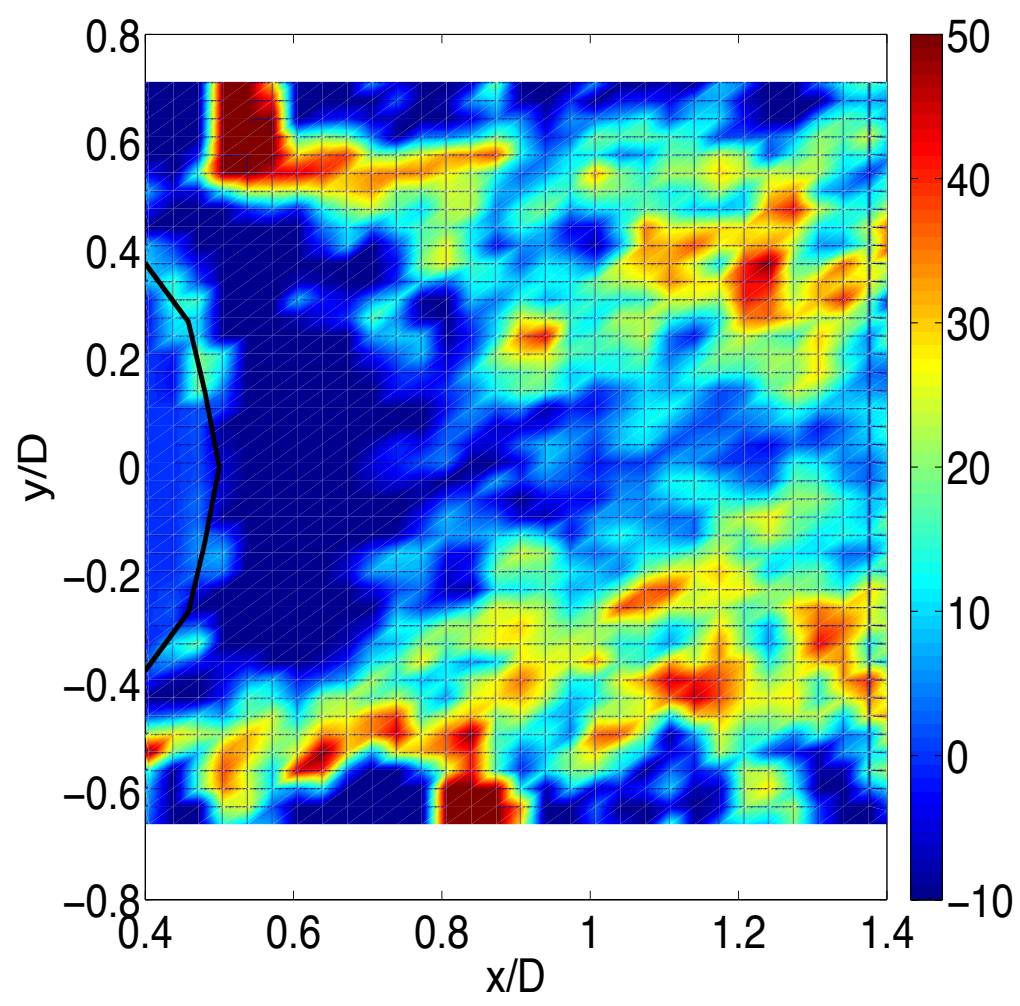

Figure 10: PIV database 1 Normalized turbulent production. 


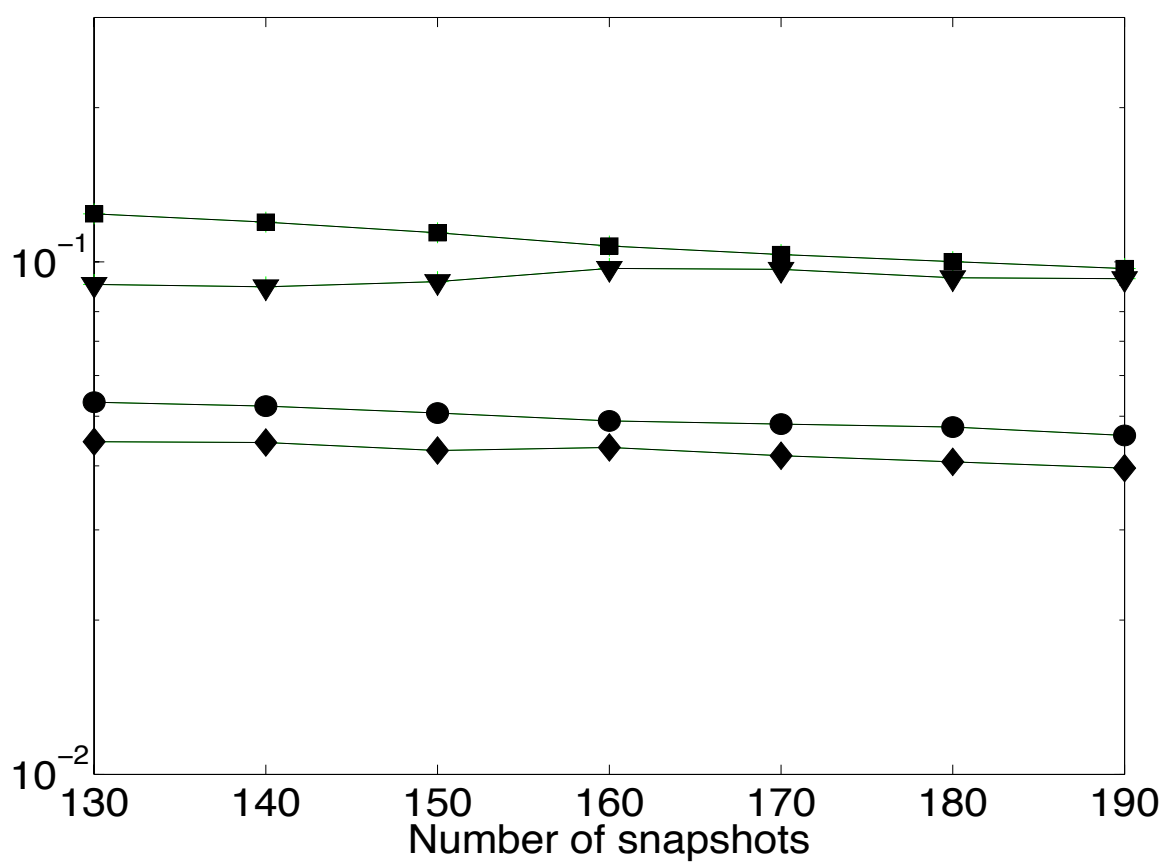

Figure 11: PIV database 1 Eigenvalues convergence as a function of the number of PIV snapshots. Mode number 1 : black square; Mode number 2 : black triangle; Mode number 3 : black cirl; Mode number 4 : black diamond.

$\lambda^{(n)}$

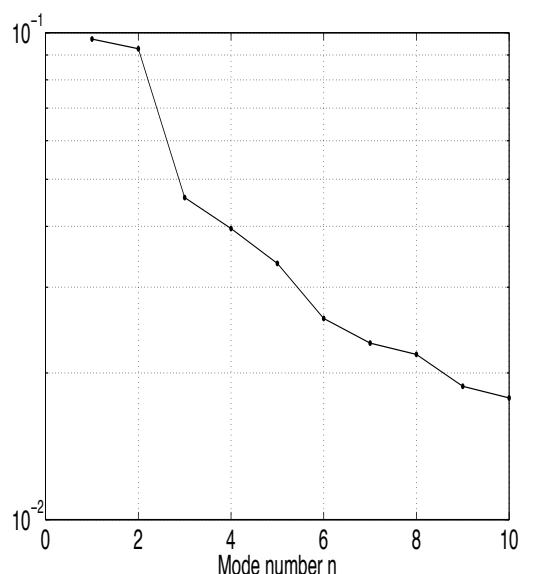

$\sum_{i=1}^{n} \lambda^{(i)} / \sum_{i=1}^{N_{\text {mod }}} \lambda^{(i)}$

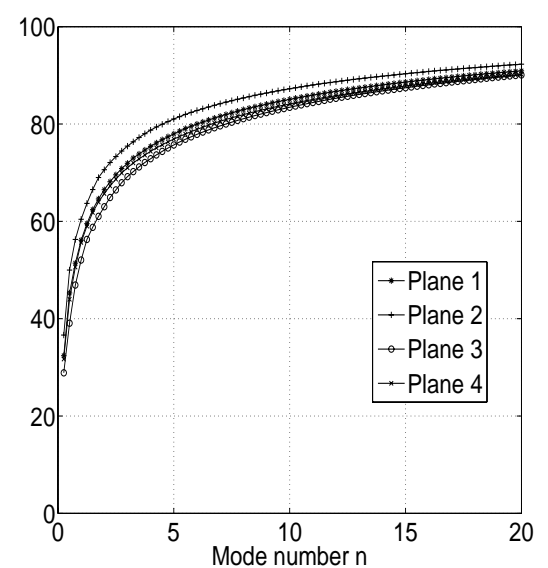

Figure 12: Left hand side: PIV database 1 Eigenvalue spectrum versus a number of POD modes. Right hand side: PIV database 2 Cumulative energy summation versus a number of POD modes. 


\begin{tabular}{|c|cccccc|}
\hline$n$ & 1 & 2 & 3 & 4 & 5 & 6 \\
\hline $100 \lambda^{(n)} / \sum_{n=1}^{N_{\text {mod }}} \lambda^{(n)}$ & 9.7 & 9.3 & 4.6 & 4 & 3.4 & 2.6 \\
\hline
\end{tabular}

Table 1: PIV database 1: Energy content of the first POD modes expressed as a percentage of the total fluctuating kinetic energy.
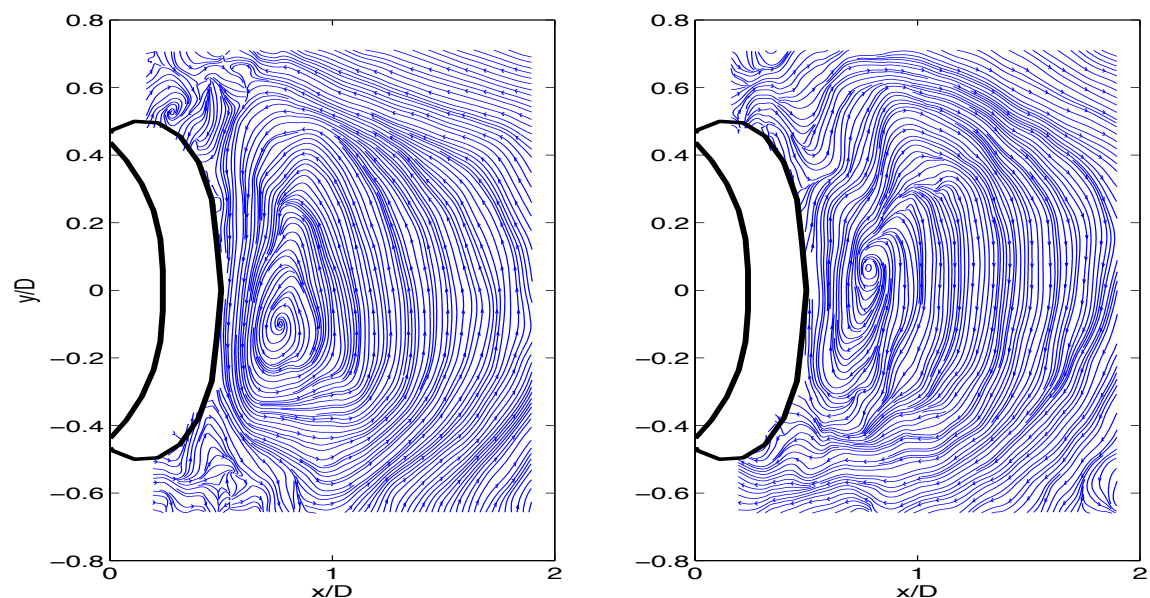

Figure 13: PIV database 1 Flow streamline topology of the first (left hand side) and the second (right hand side) POD eigenfunctions.

POD eigenfunction analysis.

Figures 13 displays the first two POD modes which are related to the convection of the vortices. These modes exhibit similar shapes. It is then not possible to define a phase angle between the first two modes as previous bluff body wake studies have shown [20,22]. This difference may be explained with the non-symmetry along the $y=0$ axis. Indeed, the flow streamline topology of the first 2 POD modes exhibits a vortex core at different $y$-locations $(x / D=0.75, y / D= \pm 0.1)$.

Figures 14 and 15 display the first and the second POD eigenfunctions respectively, obtained from the POD application in each of the four PIV planes. Even if the space domain is reduced to the upper near wake of the structure, it is observed, in the wake $(x / D>0.5)$, a shift in the $x$ direction between the first and the second POD modes. Such result can then be related to previous investigations dealing with other wake structures and the associated phase shift between the first two POD modes.

\section{POD coefficient analysis.}

The temporal coefficients $a^{(1)}(t)$ and $a^{(2)}(t)$ computed from PIV database 1, are represented in figure 16 versus time variable. These coefficients have strong regular shapes with a period which can be related to the vortex shedding phenomenon analyzed in the next section. Note that the time evolution of high order POD coefficients do not exhibit similar quasi-periodic behavior.

The first POD coefficients computed from PIV database 2 provide also similar quasi-periodic features which are associated to the large scale flow motion observed in figure 8 . 

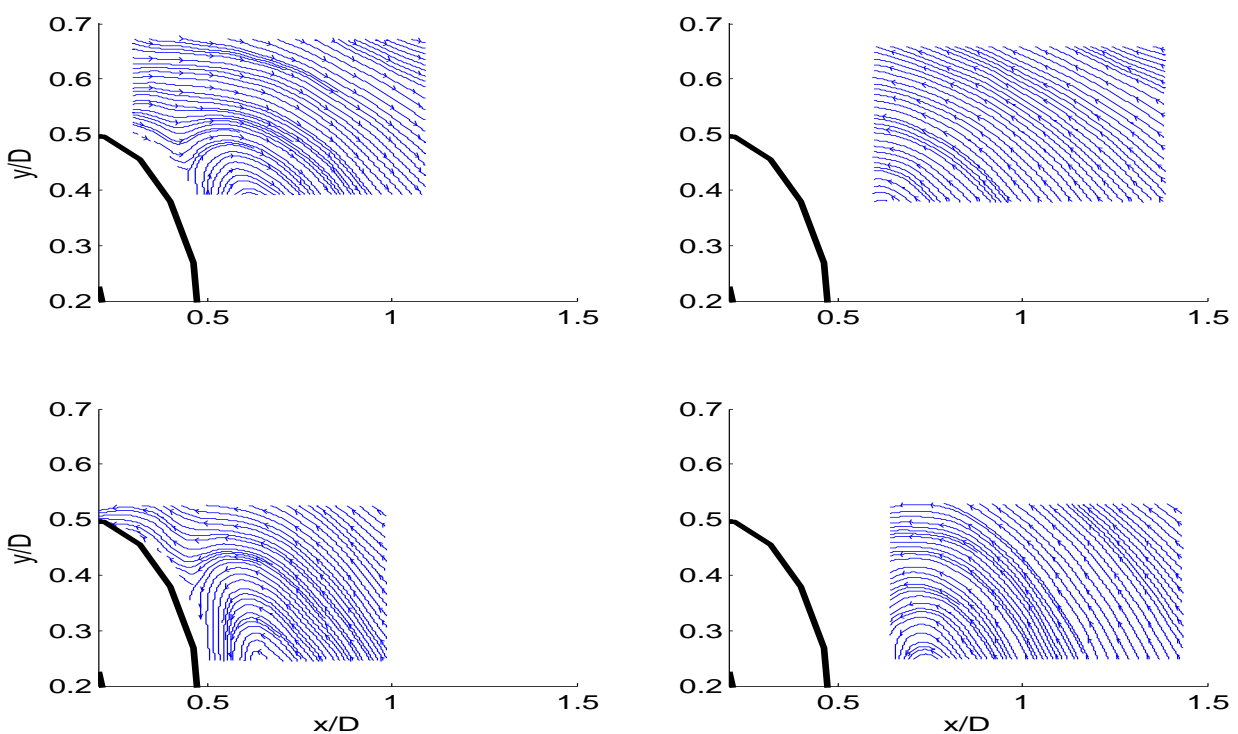

Figure 14: PIV database 2 Flow streamline topology of the first POD eigenfunction. 

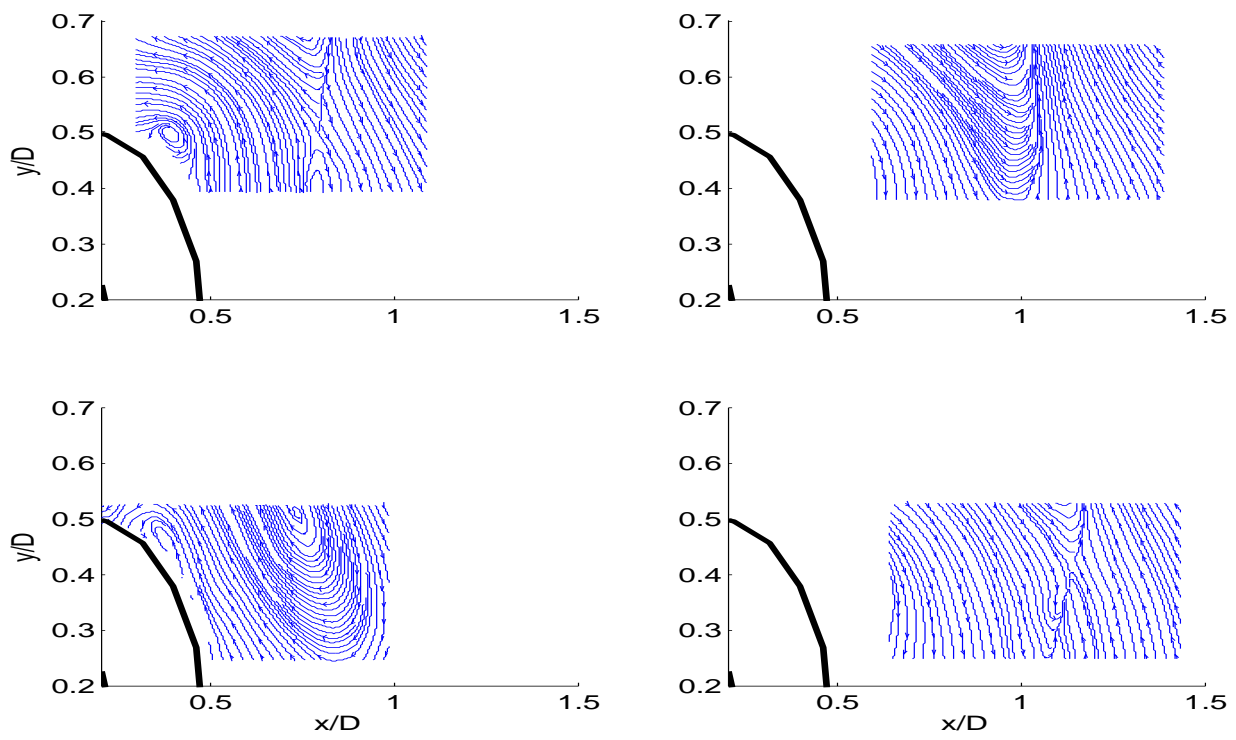

Figure 15: PIV database 2 Flow streamline topology of the second POD eigenfunction.
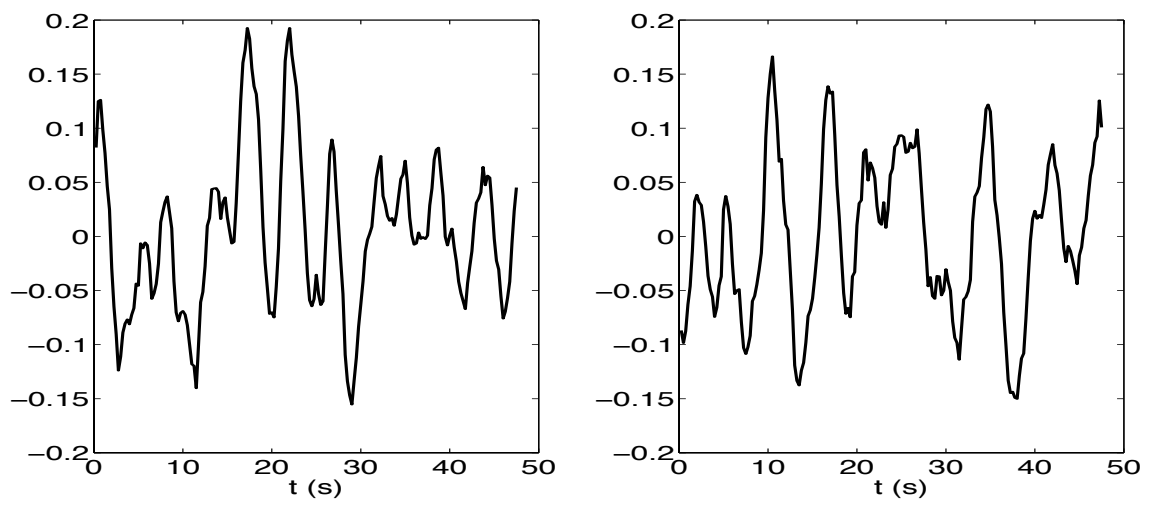

Figure 16: PIV database 1 Time evolution of the first (on the right) and second POD coefficients. 

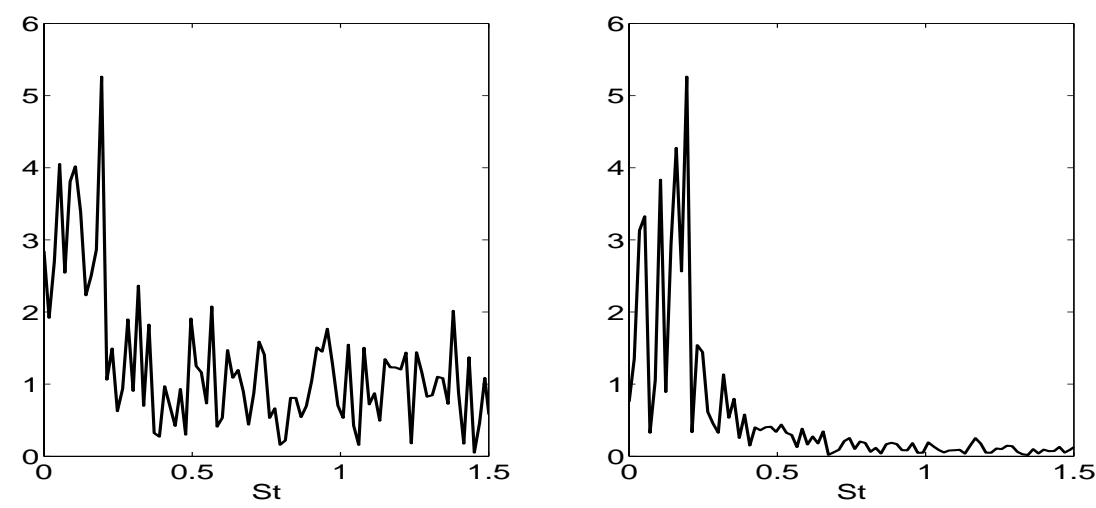

Figure 17: PIV database 1: Spectra of $u_{1}$ velocity component at a specific location: $(x / D, y / D)=(1,0.48)$. Left hand side: computed from PIV raw database. Right hand side: computed from raw data projected onto the first 2 POD modes.

\subsection{Vortex shedding characterization}

In this section, both flow configurations (fixed rigid cod-end and moving porous fishing structure) are analyzed allowing the investigation of the vortex shedding frequency as a function of the motion or not of the fishing net.

\subsubsection{Fixed rigid cod-end flow configuration}

Vortex shedding in the near wake of the rigid hemispherical structure is now investigated. To examine the passage of vortices in the near wake, the Fourier power spectrum of the longitudinal velocity component $u_{1}$ is computed. Figure 17 displays the power spectrum of $u_{1}$ computed at a discrete location in the near wake: $(x / D, y / D)=(1,0.48)$. This spectrum is plotted versus the Strouhal number:

$$
S_{t}=f D / U_{r e f}
$$

where $f$ denotes the frequency. Using raw PIV database, the spectrum exhibits a maxima peak corresponding to a Strouhal number of 0.19. This values approaches the classical one $S_{t}=0.2$ obtained in the wake of bluff bodies at high Reynolds flow [6]. Applying POD technique, the instantaneous velocity field is projected onto the first two POD modes. The power spectrum computed from the resulted velocity field is also plotted in figure 17 (right hand side). It compares quite well to the original one, showing the effectiveness of POD procedure in well extracting the passage of the large scale flow structures in the wake. Indeed, the maximum frequency peak obtained for $S_{t}=0.19$ agrees with the one obtained from raw PIV data. This peak corresponds to the classical Karman vortex frequency. In this flow configuration, the vortex shedding motion behind the rigid cod-end is well represented by a small number of POD modes. In this sense, POD procedure acts as a filtering technique for the frequency analysis.

Due to limited number of available PIV vector fields (PIV database 1$)$, this analysis can be improved by using the PIV database 2. In a similar location $(x / D, y / D)=(1,0.5)$, extracted from measurements in plane 4 , the Fourier power spectrum of the $u_{1}$ velocity component is examined. Figure 18-top presents the results deduced from raw data. The frequency peak obtained at $S_{t}=$ 0.2 and associated with the classical Von Karman frequency is retrieved. A second frequency peak is observed at $S_{t}=0.36$. The associated frequency equals to approximately twice the 

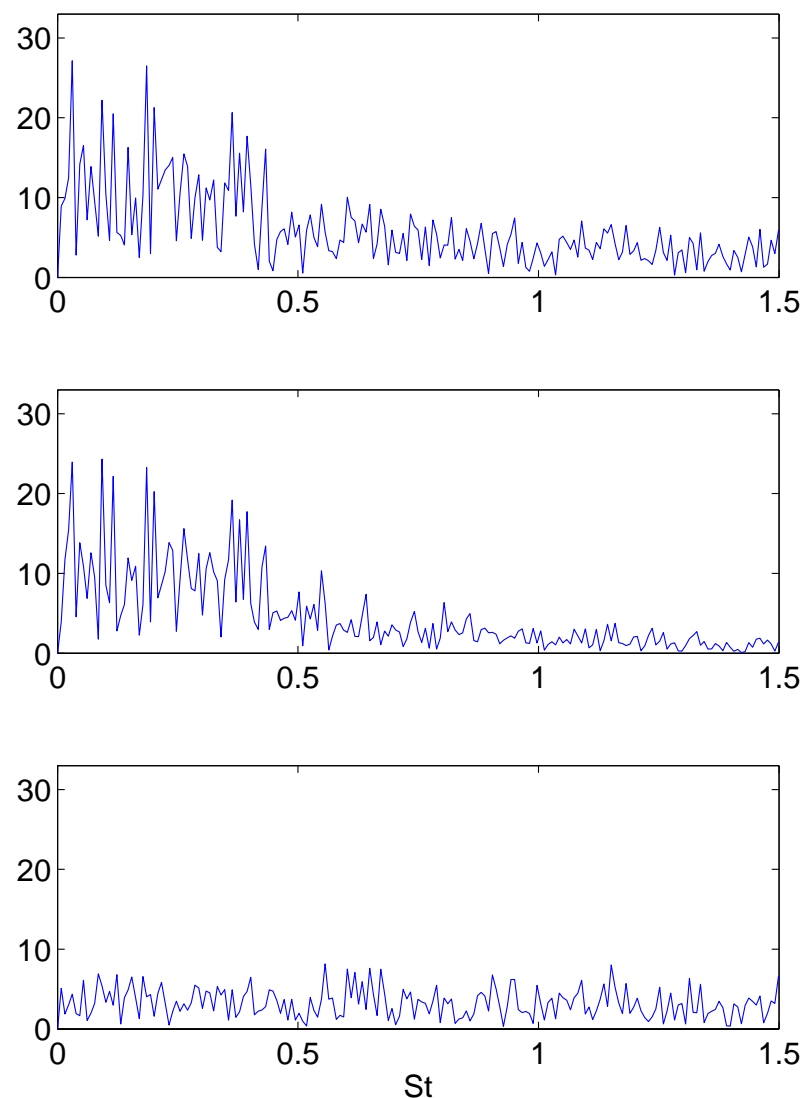

Figure 18: PIV database 2, Plane 4: Spectra of $u_{1}$ velocity component at a specific location:, $(x / D, y / D)=(1,0.5)$. Top to bottom : computed from PIV raw database; projected onto the first 5 POD modes; projected onto the residual POD modes (from 6 to $\mathrm{N}_{\mathrm{mod}_{2}}$. 
frequency of Karman structures. It then corresponds to the second harmonic of the fundamental frequency as observed previously in a flow around a circular cylinder at high Reynolds number [26]. Furthermore, applying POD technique, the power spectrum of the $u_{1}$ component projected onto the first 5 modes (containing 55\% of the total kinetic energy) is also represented on figure 18. The power spectrum of $u_{1}$ projected onto the POD residu (that is from POD mode number 6 to $N_{\text {mod }_{2}}$ ) is also given in this figure. It is observed that both frequency peaks are well detected with the first POD modes. The flow structures associated with the POD residu can be viewed as incoherent turbulent background fluctuations having a flat spectrum. Moreover, the signature of coherent flow structures is well recovered with the first POD modes.

\subsubsection{Moving porous bottom trawl configuration}

As a first investigation, the oscillatory motion of the porous cod end is examined. Indeed, in this flow configuration, the fishing net structure is free to move in the three directions. The motion along the streamwise direction is possible because of the net deformation of the trawl. Furthermore, recall that the motion along the spanwise direction, relatively small here, can not be taken into account from available 2D PIV measurements. The dominant transverse oscillation of the fishing structure is then essentially investigated. The time evolution of selected points located on the surface of the porous structure is then analyzed. Similar time evolutions are obtained in each retained point. An illustration is provided on figure 19. The associated spectral representation is also given on the right hand side of this figure.

Note that the computed spectra (associated with the moving bottom trawl configuration) given in figure 19 and also in figure 20 are not be represented in a non-dimensional form. Indeed, as it has been outlined in Sarpkaya [27], when dealing with complex flow configuration, many parameters (such as body diameter, oscillation amplitudes of the body, reduced velocity associated with these oscillations, ...) can be used for normalization. In our test case, according to the available PIV database, it is not possible to properly investigate each of these parameters, justifying the representation of the following spectra in a dimensional form.

It is then observed that the porous structure exhibits two main quasi-periodic motions. The first one associated to a weak frequency $(f=0.055 \mathrm{~Hz})$ corresponds to a quasi-periodic motion with an amplitude of an order $d / 10$. This motion has been presented on figure 19 with a black dash-line. Due to the limited number of available PIV snapshots, one has to take precautions in interpreting such oscillation motion. Indeed, only one period of signal is available. This does not permit to fully well statistically investigate such oscillation motion. The second oscillation is associated with a higher frequency of $f=0.22 \mathrm{~Hz}$. Both transverse oscillations are related to the flow instabilities and also to the whole three-dimensional free motion of the fishing net structure.

The second investigation concerns the near wake vortex shedding. Using the available TRPIV velocity data information, a spectral analysis of the longitudinal velocity component $u_{1}$ is performed. Figure 20 (left hand side) presents the $u_{1}$ power spectrum computed at a selected location: $(x / d, y / d)=(1,0.48)$. This spectrum exhibits two frequency peaks. These last ones correspond to the ones computed from the power spectra of the time evolution of the bottom trawl (see figure 19). There is a synchronization of vortex shedding with the fishing net structure oscillations. The lock-in phenomenon is then clearly exhibited.

POD procedure is now implemented to test its effectiveness in providing a low-dimensional description of the wake even in presence of the free three-dimensional motion of the fishing net structure. For such a purpose, instantaneous velocity fields are stored in the near wake domain 

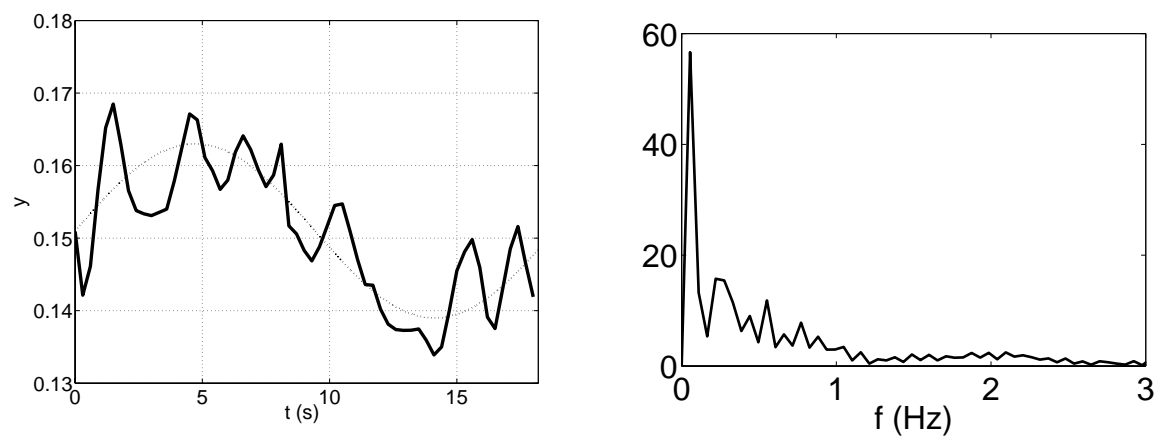

Figure 19: PIV database 3: Left hand side: Time evolution (black line) of the bottom trawl during TRPIV measurements. Dash line indicates the low frequency oscillation. Right hand side: Corresponding spectral representation.
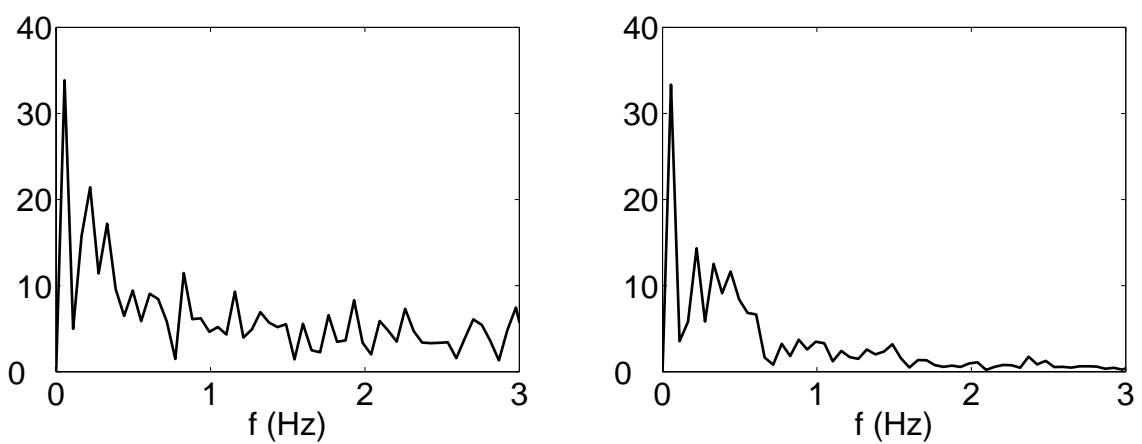

Figure 20: PIV database 3: Spectra of $u_{1}$ velocity component at a specific location $(x / d, y / d)=(1,0.48)$. Left hand side: computed from raw PIV database. Right hand side: computed from raw data projected onto the first 3 POD modes.

(dash-line plane given in figure 6). A snapshot POD is then applied to the fluctuating velocity field available onto a regular meshgrid of $n_{x}^{\prime} \times n_{y}^{\prime}=25 \times 30$ points. 272 POD eigenfunctions are obtained. The first three modes contain $32 \%$ of the total fluctuating energy. Each available instantaneous velocity field is then projected onto these first 3 POD modes. The power spectrum of this projected velocity field is then computed and presented in figure 20 (right hand side). As it has been observed in the other flow configuration, the first POD modes contains the main flow information related to the vortex shedding. Indeed, resulted spectrum exhibits both similar frequency peaks as the ones computed from raw PIV database. The first three POD modes contains the frequency information related to the oscillatory motion of the fishing net structure.

It is important to note that these last results are preliminary ones. Further investigations based on TRPIV measurements have to be performed. First, future measurements will have a sufficient time resolution and time duration ensuring a better convergence of the computed statistics. This will also permit to fully investigate not only the vortex shedding frequency but also the oscillatory motion of the fishing net structure. Second, it would be also interesting to perfom TRPIV measurements in a horizontal plane to detect the streamwise and spanwise oscillatory motion of the fishing net structure. Such TRPIV measurements are on going. 


\section{Conclusions}

Flow field measurements behind a rigid cod-end are conducted using Time Resolved PIV method. Such a method is based on an image acquisition rate allowing the time resolved sampling of the vortex shedding phenomenon. These experiments form one of the first studies in which the main space and time flow characteristics around a modelled fishing net structure are investigated. First, the main flow characteristics behind the rigid cod end structure have been determined. The Reynolds averaged field analysis shown that the wake flow of the hemispherical cod-end structure compares quite well with the flow past a cylinder or a sphere. Second the application of POD provides also some similar results than the ones previously obtained in a flow past a cylinder (or a sphere). Third, the frequency analysis demonstrated that the vortex shedding phenomenon obtained behind a rigid cod-end has some similarities with the one behind a cylinder with a Strouhal number approaching 0.2. Furthermore, it is demonstrated the effectiveness of the POD procedure to isolate the large scale flow structures (containing the most kinetic energy) from the background incoherent fluctuations. POD is shown to be robust to act as a filter for the frequency analysis. Indeed, a low order representation composed of the mean flow and the first POD eigenfunctions provides a good description of the coherent wake motion, enabling the identification of the major characteristics of the vortex shedding process.

Moreover, preliminary TRPIV measurements have been performed around a moving bottom trawl. Based on a vortex shedding frequency analysis, the near wake vortex shedding is found to synchronize with the oscillation of the fishing net structure. POD investigation confirms the potential of POD technique in isolating the vortex shedding flow structures with the aid of the first POD modes. These preliminary results seem promising for future investigations of the threedimensional flow field around a fishing net structure able to free moving.

A better understanding of the physical phenomenon is a first step towards the knowledge of the impact of this kind of porous structure that can have both on the flow instabilities and on the overall drag generation. Indeed, the fluid - structure interaction effects can reach to the perturbation of the flow in term of ambient turbulence intensity and velocity profiles. In return, the geometry of the trawl can then be modified. Some alterations on the different boundary layer developments will be produced by these structural evolutions. This leads to the necessity to conduct fluid - structure characterisation on less specific trials in order to be able afterwards to correct the locally hydrodynamic loads used to calculate an entire trawl. However, these experimental results will be used to validate numerical development conducted to simulate the behaviour of both bottom and pelagic trawls.

\section{Acknowledgements}

The authors would like to gratefully acknowledge the French Ministry of Agriculture and Fisheries and the European Community for their financial support during the HydroPeche project monitored by IFREMER (French Research Institute for Exploitation of the Sea). The authors also would like to acknowledge JV. Facq and B. Gaurier for their implication in the experimental work.

\section{References}

[1] E. Dahm, H. Wienbeck, C. West, J. Valdemarsen, F. O'Neill, On the influence of towing speed and gear size on the selective properties of bottom trawls, Fisheries Research 55 (2002) 103-119. 
[2] D. Priour, Numerical optimisation of trawls design to improve their energy efficiency, Fisheries Research 98 (2009) 40-50.

[3] F. O’Neill, L. Knudsen, D. Wileman, S. McKay, Cod-end drag as a function of catch size and towing speed, Fisheries Research 72 (2005) 163-171.

[4] M. Pashen, W. Köpnick, H. Winkel, Model tests of various cod-end constructions - the key for an explanation of selectivity characterisation ?, in: Contributions of theory of fshing gears and related marine systems, Vol. 2, Ingo Koch-Verlag \& Co. KG, Rostock., 2002.

[5] D. Priour, F. O’Neil, A. Sala, P. Chevallier, B. Hermann, Premecs-ii final report: Development of predictive model cod-end selectivity, Tech. rep. (2006).

[6] C. Williamson, R. Govardhan, Vortex induced vibrations, Ann. Rev. Fluid Mech. 36 (2004) 413-455.

[7] R. Govardhan, C. Williamson, Vortex-induced vibrations of a sphere, J. Fluid Mech. 531 (2005) 11-47.

[8] G. Pichot, G. Germain, D. Priour, On the experimental study of the flow around a fishing net, Eur. J. Mech. B/Fluids (2009) 103-116.

[9] F. O’Neill, T. O'Donoghue, The fluid dynamic loading on catch and the geometry of trawl cod-ends, Proc. R. Soc. Lond. A (453) (1997) 1631-1648.

[10] L. Meyler, Simulation of net structures hydrodynamic fields, 'Modelling and Simulation', InTech Education and Publishing, G. Petrone and G. Cammarata, 2008, Ch. 16, pp. 261-282.

[11] O. Patursson, M. Robinson Swift, I. Tsukrov, K. Simonsen, K. Baldwin, D. Fredriksson, C. B., Development of a porous media model with application to flow through and around a net panel, Ocean Eng. 37 (2010) 314-324.

[12] R. Adrian, Twenty years of particle image velocimetry, Exp. Fluids 29 (2005) 159-169.

[13] P. Holmes, J. Lumley, G. Berkooz, Turbulence, coherent structures, dynamical systems and symmetry., Cambridge monograph on mechanics eds., 1996.

[14] P. Druault, J. Delville, Representation of the spatial correlation tensor of the velocity in free turbulent flows, C.R.A.S Serie II-B 328 (2) (2000) 135-141.

[15] P. Druault, J. Delville, J. Bonnet, Experimental 3d analysis of the large scale behaviour of a plane turbulent mixing layer, Flow Turb. Comb. 74 (2) (2005) 207-233.

[16] P. Druault, C. Chaillou, Use of proper orthogonal decomposition for reconstructing the $3 \mathrm{~d}$ in-cylinder mean-flow field from piv data, C.R. Mecanique 335 (1) (2007) 42-47.

[17] P. Druault, P. Guibert, F. Alizon, Use of proper orthogonal decomposition for time interpolation from PIV data, Exp. Fluids 39 (2005) 1009-1023.

[18] J. Lumley, The structure of inhomogeneous turbulent flows, in: Yaglom, Tatarsky (Eds.), Atm. Turb. and Radio wave Prop., 1967, pp. 166-178.

[19] L. Sirovich, Turbulence and the dynamics of coherent structures. part i: Coherent structures, Q. Appl. Math XLV (1987) 561-571.

[20] B. Van Oudheusden, F. Scarano, N. Van Hinsberg, D. Watt, Phase-resolved characterization of vortex shedding in the near wake of a square-sectioncylinder ar incidence, Exp. Fluids 39 (2005) 86-98.

[21] S. Roudnitzky, P. Druault, P. Guibert, Proper orthogonal decomposition of in-cylinder engine flow into mean component, coherent structures and random gaussian fluctuations, J. of Turbulence 7 (70) (2006) 1-19.

[22] R. Perrin, M. Braza, E. Cid, S. Cazin, A. Barthet, A. Sevrain, C. Mockett, F. Thiele, Obtaining phase averaged turbulence properties in the near wake ofa circular cylinder at high reynolds number using pod, Exp. Fluids 43 (2005) 341-355.

[23] V. A. Huera-Huarte FJ, Vortex modes in the wake of an oscillating long flexible cylinder combining pod and fuzzy clustering, Exp. Fluids 48 (2010) 999-1013.

[24] M. Braza, R. Perrin, Y. Hoarau, Turbulence properties in the cylinder wake at high reynolds numbers, J. Fluids Struct. 22 (2006) 757-771.

[25] A. Santa Cruz, L. David, J. Pecheux, A. Texier, Characterization by proper-orthogonal-decomposition of the passive controlled wake flow downstream of a half cylinder, Exp. Fluids 39 (4) (2005) 730-742.

[26] S. Rajagopalan, R. Antonia, Flow around a cylinder-structure of the near wake shear layer, Exp. Fluids 38 (2005) 393-402.

[27] A. Sarpkaya, A critical review of the intrinsic nature of vortex-induced vibrations, J. Fluids Struct. 19 (2004) 389-447. 